\title{
Zhenglu Jiang \\ Dynamical modelling of the elliptical galaxy NGC 2974
}

Received: November 3, 2018/ Accepted: date

\begin{abstract}
In this paper we analyse the relations between a previously described oblate Jaffe model for an ellipsoidal galaxy and the observed quantities for NGC 2974, and obtain the length and velocity scales for a relevant elliptical galaxy model. We then derive the finite total mass of the model from these scales, and finally find a good fit of an isotropic oblate Jaffe model by using the Gauss-Hermite fit parameters and the observed ellipticity of the galaxy NGC 2974. The model is also used to predict the total luminous mass of NGC 2974, assuming that the influence of dark matter in this galaxy on the image, ellipticity and Gauss-Hermite fit parameters of this galaxy is negligible within the central region, of radius $0.5 R_{\mathrm{e}}$.
\end{abstract}

Keywords celestial mechanics $\cdot$ stellar dynamics $\cdot$ galaxies

\section{Introduction}

Galaxies are usually modelled in terms of their morphology, surface luminosity profile, mass-light ratio, velocity dispersion profile and the line-of-sight velocity distribution curve (often termed the rotation curve). Recently, dynamical modelling of the ellipsoidal galaxy NGC 2974 was performed by Cinzano and van der Marel (1994), based on the Gauss-Hermite fit parameters, i.e. the rotation velocities $\hat{v}$, velocity dispersions $\hat{\sigma}$ and deviations of the velocity profiles from a Gaussian, as quantified by the Gauss-Hermite moments $h_{3}$ and $h_{4}$. A similar modelling of the ellipsoidal galaxy M32 was given by Qian et al. (1995). After that, Baes and Dejonghe (2004) provided a completely analytical class of dynamical models for spherical galaxies and bulges with central black holes. Baes et al. (2005) also studied the dynamical structure of isotropic spherical galaxies with a central black hole. Some dynamical models of luminous and dark matter for 17 early-type galaxies were recently presented by Thomas et al. (2007). Previously a number of more extensive studies have been published (e.g. Gerhard 1993a,b; van der Marel \& Franx 1993; Bender et al. 1994; Gerhard et al. 1998; Kronawitter et al. 1999; Saglia et al. 2000; Halliday et al. 2001). In particular, the works of Emsellem, Goudfrooij and Ferruit (2003) and Krajnović et al. (2005) are very thorough investigations of NGC 2974. The former shows a two-arm gaseous spiral in the inner 200 pc of the early-type galaxy NGC 2974 by fitting the TIGER and long-slit stellar kinematic data of the galaxy with a dynamical model, not requiring the addition of a disc or a central dark mass. For their dynamical model, a numerical two-integral distribution function was derived from a multi-Gaussian expansion mass model by using the Hunter-Qian (1993) algorithm. The paper of Krajnović et al. presents a very thorough analysis of NGC 2974 using completely different data and methodology. Krajnović et al. first obtained a multi-Gaussian expansion mass density, fitting simultaneously the ground-based I-band image and dust-corrected PC part of the WFPC2/F814 photometry of this galaxy; they then constructed a three-integral model from the recovery of the mass density using the Schwarzschild (1979) orbit-superposition method; finally, they provided a dynamical modelling of stars and gas in this galaxy by use of the Gauss-Hermite fit parameters. In this paper we again model dynamically NGC 2974, by employing distribution functions from self-consistent density-potential pairs of an oblate Jaffe model (Jiang 2000) and using the Gauss-Hermite fit parameters and the ellipticity (Sandage and Tammann 1981). The line-of-sight velocity distribution curves are calculated from the distribution functions for the oblate Jaffe model and quantified by its Gauss-Hermite fit parameters. The derived Gauss-Hermite fit 
parameters for the oblate Jaffe model correspond very well to those of NGC 2974. The model is also applied to predict the total luminous mass of NGC 2974, since in the region of a radius of $0.5 R_{\mathrm{e}}\left(R_{\mathrm{e}}=33.7\right.$ arcsec $)$ the dynamical influence of the dark halo of NGC 2974 is negligible (cf. Cinzano and van der Marel 1994).

As indicated above, a distribution function can be derived from a mass density by either the Hunter-Qian algorithm or the Schwarzschild method. As in some other published studies (e.g. Qian et al. 1995; Emsellem, Goudfrooij and Ferruit 2003), we obtain below a distribution function using the Hunter-Qian (1993) algorithm. It is worth mentioning that the first step of our dynamical model is to give directly the oblate Jaffe mass model by using only the galactic ellipticity in order to mimic the image of NGC 2974. This is different from the approach used by all the earlier works mentioned above.

NGC 2974 has been studied frequently (e.g. Gallouet et al. 1975; Sandage and Tammann 1981; DemoulinUlrich et al. 1984; Forman et al. 1985; Canizares et al. 1987; Davies et al. 1987; de Vauouleurs et al. 1991). It appears to be completely isolated (Kim et al. 1988), is classified as E4 by Sandage and Tammann (1981), and has a regular distribution of gas (Kim et al. 1988) and a dark halo (Cinzano and van der Marel 1994). The total mass of NGC 2974 within a galacto-centric radius of $13 \mathrm{kpc}$ is estimated as $3.8 \times 10^{11} h^{-1} \mathrm{M}_{\odot}$ by $\mathrm{Kim}$ et al. (1988), and its total luminous mass as $1.2 \times 10^{11} h^{-1} \mathrm{M}_{\odot}$ (Cinzano and van der Marel 1994), where $h$ is Hubble's constant. A range of a factor of 2 is given for $h$ [ $h$ is in units of $100 \mathrm{kms}^{-1} \mathrm{Mpc}^{-1}$ and is thought to lie in the range $0.5 \sim 1$ ]. Since the first results of the WMAP mission have been made public, we now know the constant $h$ with a much smaller uncertainty (Bennett 2003). Cinzano and van der Marel (1994) also showed that the total mass of the dark halo is about $1.8 \times 10^{11} h^{-1} \mathrm{M}_{\odot}$. Thus, according to this estimate, the total mass of NGC 2974 is $3.0 \times 10^{11} h^{-1} \mathrm{M}_{\odot}$, which is obviously smaller than that given by Kim et al., mentioned above. The difference appears to be due mainly to differences in the adopted distance and in minor details of the model (van der Marel, private communication). Kim (1988) showed that the optically measured heliocentric velocity of NGC 2974 is $1924 \mathrm{kms}^{-1}$ according to the optical definition $v=c \Delta \lambda / \lambda_{0}$, and that the distance to NGC 2974 is given in terms of the value of Hubble's constant and is $22.5 \mathrm{~h}^{-1} \mathrm{Mpc}$ in terms of the Virgocentric flow model of Aaronson et al. (1982) [it is $19.25 \mathrm{~h}^{-1} \mathrm{Mpc}$ based on the Hubble law]. The rotational velocity of NGC 2974 is about $360 \mathrm{kms}^{-1}$ (Kim et al. 1988) and its central velocity dispersion is about $222 \mathrm{kms}^{-1}$ (Kim et al. 1988; Cinzano and van der Marel 1994). Gauss-Hermite fit parameters of NGC 2974 along the major and minor axes have been derived independently by Bender et al. (1994) and Cinzano and van der Marel (1994).

We use a stellar dynamical galaxy model to calculate Gauss-Hermite fit parameters $\left(\hat{\sigma}, \hat{v}, h_{3}, h_{4}\right)$ as a function of the dimensionless position variable $x^{\prime}$ (measured from the potential centre along the major axis). The physical parameters in the models can then be related to the physical dimensions of the observed galaxy with the help of the length and velocity scales determined by minimizing a certain function. This function can be constructed in the physical domain by use of dimensionless Gauss-Hermite fit parameters $\left(\hat{\sigma}, \hat{v}, h_{3}, h_{4}\right)$, together with the corresponding observations of an elliptical galaxy, by using a weighted least squares method. An isotropic model can then be selected from this class of axisymmetric elliptical galaxy models by analysing the image and ellipticity of an elliptical galaxy (in our case NGC 2974). It is known from Fig. 4 in Sect. 5 that the Gauss-Hermite fit parameters $\left(\hat{\sigma}, \hat{v}, h_{3}, h_{4}\right)$ of the model can fit the corresponding observations of the elliptical galaxy NGC 2974 in terms of the above least squares method. Finally, the total mass of the best fit model of NGC 2974 can be derived from the length and velocity scales obtained by comparing the Gauss-Hermite fit parameters $\left(\hat{\sigma}, \hat{v}, h_{3}, h_{4}\right)$ with the corresponding observations of NGC 2974.

The models we give here are ideal, in that effects from the surrounding environment are not considered. The more a galaxy is affected by neighbouring objects, the less well the observations can be expected to be fitted by such an ideal model. A galaxy needs to be quite close to us for adequate observations to be available to determine such effects. It is not obvious that the best fitting model will be a flattened Jaffe model, but here we restrict ourselves to the latter. We first construct the oblate Jaffe model and search for a fit. Obviously, the existence of a good fit is not guaranteed but it turns out that one can be found. It is possible that other such 'suitable' galaxies amenable to this analysis exist but, up to now, no suitable galaxy other than NGC 2974 has been found.

This paper is arranged as follows. We first introduce oblate Jaffe models and Gauss-Hermite fit parameters in Sect.2. Then, in Sect.3, we mainly analyse relations between the oblate Jaffe model and observed quantities, in order to obtain the length and velocity scales of the oblate models. Having derived the finite total mass of the models from these scales in Sect. 4, we can then, based on the Gauss-Hermite fit parameters and the ellipticity of an elliptical galaxy NGC 2974, find an isotropic oblate Jaffe model in Sect.5 that is a good fit. The derived total mass of the isotropic model only reflects the total luminous mass of NGC 2974. This is because we can neglect the influence of dark matter in NGC 2974 on the image, ellipticity and Gauss-Hermite fit parameters of the observed galaxy within the radius of $0.5 R_{\mathrm{e}}$. Our work is summarized in Sect.6 


\section{Oblate Jaffe Models and Gauss-Hermite Fit Parameters}

Jiang (2000) defined a class of oblate axisymmetric elliptical systems with the potential-density pairs

$$
\Phi\left(R^{2}, z\right)=\frac{G M}{r_{J}} \ln \left(\frac{\sqrt{R^{2}+\left(\sqrt{z^{2}+c^{2}}+d\right)^{2}}}{\sqrt{R^{2}+\left(\sqrt{z^{2}+c^{2}}+d\right)^{2}}+r_{J}}\right)
$$

and

$$
\rho\left(R^{2}, z\right)=\frac{M}{4 \pi r_{J}} \frac{c^{2} d r_{J} \tau^{3}+r_{J}^{2} \tau^{2}\left(Y^{3}+c^{2} d\right)+\left(3 \tau+2 r_{J}\right) c^{2} r_{J} Y(Y+d)^{2}}{\tau^{4}\left(\tau+r_{J}\right)^{2} Y^{3}},
$$

where $R$ and $z$ are two of three cylindrical coordinates $(R, z, \phi), M, c, d$ and $r_{J}$ are positive constants, $G$ is the gravitational constant, $Y=\sqrt{z^{2}+c^{2}}$ and $\tau=\sqrt{R^{2}+(Y+d)^{2}}$. Here, these systems are obtained by flattening the spherical Jaffe (1983) model and they are oblate, and so they are called oblate Jaffe models. Some more general extensions of the spherical Jaffe model have been given by Jiang and other coauthors (Jiang and Moss 2002; Jiang, Fang and Moss 2002). It is worth mentioning that the spherical Jaffe model is a special case of a family of spherical $\gamma$ models (Kuzmin, Veltmann, Tenjes 1986; Dehnen 1993; Saha 1993; Tremaine et al. 1994) which have some similar flattening properties (Jiang, Fang, Liu and Moss 2002; Jiang and Ossipkov 2006; Ossipkov and Jiang 2007a,b). In the literature, there are also other spherical models (e.g. Veltmann 1961, 1965, 1979, 1981; Kuzmin and Veltmann 1967a,b, 1973; Ossipkov 1979a,b; Binney and Tremaine 1987) and generalized systems with three-dimensional gravitational potentials (e.g. Kutuzov and Ossipkov 1981).

It is known that the density (2) at large distances decays radially like $r^{-4}$, except on the major axis, and as $r^{-3}$ on the major axis, where $r$ is one of three spherical coordinates $(r, \theta, \phi)$. The central ratio $\alpha$ of isodensity contour $R$-axis to $z$-axis (Jiang 2000) is given by

$$
\alpha^{2}=\frac{(c+d)\left(15 c^{3}+27 c^{2} d+15 c d^{2}+3 d^{3}+10 c^{2} r_{J}+9 c d r_{J}+3 d^{2} r_{J}\right)}{c^{2}(5 c+d)\left(3 c+3 d+2 r_{J}\right)}
$$

The total mass of the model is finite and equal to $M$.

Jiang (2000) also proved that the form of the potentials allows their two-integral even distribution functions to be calculated from the self-consistent density-potential pairs of the above oblate Jaffe model by using a modification of Hunter and Qian's algorithm (Hunter and Qian 1993; Qian et al. 1995). Once the two-integral even distribution functions have been obtained, the distribution functions $f\left(\varepsilon, L_{z}\right)$ can be found by using the maximum entropy principle (Dejonghe 1986), where $\varepsilon$ and $L_{z}$ are the relative energy and the $z$-axis angular momentum respectively. For oblate stellar models, other distribution functions can also be found depending on whether isotropy or anisotropy (Binney and Tremaine 1987) is assumed. Recently, some new formulae for distribution functions for both spherical and axisymmetric galaxies have been presented by Jiang and Ossipkov (2007a,b,c); but they do not seem applicable to the oblate Jaffe models.

In order to understand the Gauss-Hermite fit parameters clearly, we first assume that a galaxy is in the frame of reference $(x, y, z)$ with mass density given by Eq. (2), and $O x, y, z$ are its principal axes. Obviously, $R=\sqrt{x^{2}+y^{2}}$. Then, without loss of generality, we take the viewing direction to be in the $(y, z)$ plane and project the galaxy along the line of sight direction, which is taken as the $z^{\prime}$-axis, with an inclination angle $\varphi$ to the $z$-axis, onto the perpendicular $\left(x^{\prime}, y^{\prime}\right)$ plane. Thus a new frame of reference $\left(x^{\prime}, y^{\prime}, z^{\prime}\right)$ is formed by the sky plane $\left(x^{\prime}, y^{\prime}\right)$ and the line of sight which is the $z^{\prime}$-axis; the frames of reference satisfy the following relations

$$
x=x^{\prime}, y=y^{\prime} \cos \varphi-z^{\prime} \sin \varphi, z=y^{\prime} \sin \varphi+z^{\prime} \cos \varphi .
$$

When the galaxy is observed at an inclination angle $\varphi$, the projected surface density $\Sigma\left(x^{\prime}, y^{\prime}\right)$ at any point $\left(x^{\prime}, y^{\prime}\right)$ on the sky plane is

$$
\Sigma\left(x^{\prime}, y^{\prime}\right)=\int_{-\infty}^{+\infty} \rho\left(\left[x^{\prime}\right]^{2}+\left[y^{\prime} \cos \varphi-z^{\prime} \sin \varphi\right]^{2}, y^{\prime} \sin \varphi+z^{\prime} \cos \varphi\right) d z^{\prime}
$$

Let $v_{\|}$and $\sigma_{\|}$be the line-of-sight velocity and velocity dispersion respectively. The components of velocity directed along $\left(x^{\prime}, y^{\prime}\right)$ and the line of sight $z^{\prime}$ can be obtained as follows:

$$
\begin{aligned}
& v_{x^{\prime}}=\left(x v_{R}-y v_{\phi}\right) / R, v_{y^{\prime}}=\left(y v_{R}+x v_{\phi}\right) \cos \varphi / R+v_{z} \sin \varphi, \\
& v_{\|} \equiv v_{z^{\prime}}=-\left(y v_{R}+x v_{\phi}\right) \sin \varphi / R+v_{z} \cos \varphi
\end{aligned}
$$


The line-of-sight velocity distribution curve of any distribution function can be given by evaluating a triple integral of the distribution function $f\left(\varepsilon, L_{z}\right)$ with respect to two velocity variables (say, $v_{x^{\prime}}$ and $v_{y^{\prime}}$ ) and one position variable (say, $z^{\prime}$ ) when $\varepsilon$ and $L_{z}$ are expressed as functions of six variables (say, $v_{x^{\prime}}, v_{y^{\prime}}, v_{z^{\prime}}, x^{\prime}, y^{\prime}$ and $\left.z^{\prime}\right)$ in the phase space. Without loss of generality, we consider the normalized line-of-sight velocity distribution curve

$$
l_{0}\left(v_{\|}, x^{\prime}, y^{\prime}\right)=L_{0}\left(v_{\|}, x^{\prime}, y^{\prime}\right) / \Sigma\left(x^{\prime}, y^{\prime}\right)
$$

with

$$
L_{0}\left(v_{\|}, x^{\prime}, y^{\prime}\right)=\iiint f\left(\varepsilon, L_{z}\right) d v_{y^{\prime}} d v_{x^{\prime}} d z^{\prime},
$$

where the triple integral is to be performed over all the physical values determined by the potential (see, e.g., Evans 1994).

On the other hand, any line-of-sight velocity distribution curve can be quantified by a Gauss-Hermite series with its Gauss-Hermite coefficients $h_{n}$, which can parameterise the velocity distribution curve to carry most of information on the shape of the velocity distribution curve (van der Marel and Franx 1993). $l_{0}\left(v_{\|}, x^{\prime}, y^{\prime}\right)$ is indeed approximated by the following Gauss-Hermite series:

$$
l\left(v_{\|}, x^{\prime}, y^{\prime}\right)=\hat{\gamma} \hat{\sigma}^{-1} \sum_{n=0}^{\infty} h_{n} u_{n}(w)
$$

with

$$
h_{n} \equiv h_{n}\left(x^{\prime}, y^{\prime}\right)=\frac{(4 \pi)^{\frac{1}{2}}}{\hat{\gamma}} \int_{-\infty}^{\infty} d v_{\|} l_{0}\left(v_{\|}, x^{\prime}, y^{\prime}\right) u_{n}(w)(n=0,1,2,3, \cdots) \text {, }
$$

and

$$
u_{n}(w)=\left(2^{n+1} \pi n !\right)^{-\frac{1}{2}} H_{n}(w) e^{-\frac{w^{2}}{2}} .
$$

Here $w=\left(v_{\|}-\hat{v}\right) / \hat{\sigma}$ and $H_{n}(w)(n=0,1,2, \cdots)$ are the standard Hermite polynomials. Thus the Gauss-Hermite coefficients $h_{n}$ can be used to explain the properties of the line-of-sight velocity distribution curve of the distribution function of stars in any elliptical galaxy. $h_{0}$ represents a Gaussian profile, and odd and even Gauss-Hermite coefficients $h_{n}$ describe asymmetric and symmetric deviations from a Gaussian profile respectively. $\hat{v}$ is called the mean radial or streaming velocity, and $\hat{\sigma}$ the velocity dispersion (van der Marel and Franx 1993). In fact, the quantities $\hat{v}$ and $\hat{\sigma}$ are two free parameters and differ from the line-of-sight velocity $v_{\|}$and dispersion $\sigma_{\|} \cdot \hat{\gamma}$ is also a free parameter, in addition to $\hat{v}$ and $\hat{\sigma}$. The parameter $\hat{\gamma}$ is called the line strength (van der Marel and Franx 1993).

The Gaussian best fit to the velocity distribution curve is such that $h_{0}=1$ and $h_{1}=h_{2}=0$ with an additional condition that the fit parameter $\hat{\gamma}$ is usually around 1 . The Gaussian best fit parameters $\left(\hat{v}, \hat{\sigma}, \hat{\gamma}, h_{n}\right)$ can be calculated enabling comparison with the corresponding observations of elliptical galaxies. When the Gaussian best fit parameters $\left(\hat{v}, \hat{\sigma}, \hat{\gamma}, h_{n}\right)$ are obtained, improved estimates for the velocity moments can also be given and so the Gauss-Hermite coefficients $h_{n}$ are also termed the Gauss-Hermite moments. Since $h_{0}-1=h_{1}=h_{2}=0$ and $\hat{\gamma} \simeq 1$ for the above Gaussian best fit, the other four Gaussian best fit parameters $\left(\hat{v}, \hat{\sigma}, h_{3}, h_{4}\right)$ determine principally the velocity distribution curve, and thus they are usually called the Gauss-Hermite fit parameters. In fact, once it is assumed that $h_{0}=1$, we can regard $\hat{\gamma}$ as a function of the two parameters: $\hat{v}$ and $\hat{\sigma}$, that is, we know from (11) that

$$
\hat{\gamma}=\sqrt{2} \int_{-\infty}^{\infty} d v_{\|} l_{0}\left(v_{\|}, x^{\prime}, y^{\prime}\right) e^{-\frac{w^{2}}{2}}
$$

which gives that $0<\hat{\gamma} \leq \sqrt{2}$ for each point $\left(x^{\prime}, y^{\prime}\right)$. 


\section{Relations between the Model and the Observed Variables}

In order to apply the oblate models to analyse a galaxy, we first have to know the relations between the model variables $\left(c, d, r_{J}\right)$ of Eq. (1) (or (2) ) and the physical dimensions of the observed galaxy. The stellar dynamical model takes $r_{J}$ and $u=\left(G M / r_{J}\right)^{1 / 2}$ as units of length and velocity, respectively. Thus, in order to relate $\left(c, d, r_{J}\right)$ in expression (11) to the physical units of the galaxy, we first have to change $\left(c, d, r_{J}\right)$ into dimensionless parameters $(\hat{c}, \hat{d})$, assuming that the unit of length is $r_{J}$ [i.e. using a transformation $\hat{c}=c r_{J}, \hat{d}=d r_{J}$ ]. Then a stellar model with dimensionless parameters $(\hat{c}, \hat{d})$ can be obtained [that is, dimensionless parameters $(\hat{c}, \hat{d})$ can be determined from the relation [3] ] in terms of the image and ellipticity of the galaxy. Finally, the length and velocity scales $r_{J}$ and $u$ can be determined by comparing the Gauss-Hermite parameters of the model with those of NGC 2974, using a weighted least squares method. This comparison is made by minimizing a function constructed below in terms of the weighted least squares method. Thus we can relate the physical parameters $\left(c, d, r_{J}\right)$ of the models to the physical scales of the galaxy.

Assume that $\left(\hat{\sigma}, \hat{v}, h_{3}, h_{4}\right)$ are Gauss-Hermite fit parameters, as functions of the dimensionless position variable $x^{\prime}$ measured from the potential centre along the major axis for the dimensionless model with variables $(\hat{c}, \hat{d})$. Then $\left(\hat{\sigma}, \hat{v}, h_{3}, h_{4}\right)$ are also dimensionless. Using the linear relation

$$
r=r_{J} x^{\prime}, v=u \hat{v}, \sigma=u \hat{\sigma} .
$$

we can relate the physical parameters of the models with the physical dimensions of the galaxy.

The observations corresponding to the Gauss-Hermite fit parameters $\left(\sigma, v, h_{3}, h_{4}\right)$ are functions of the distance $r$ from the galactic centre. Generally, 1 arcsec is chosen as the unit of the distance $r$ and $1 \mathrm{kms}^{-1}$ as the unit of velocity. The dimensionless model variables $\hat{\sigma}$ and $\hat{v}$ are functions of dimensionless position $x^{\prime}$. For the oblate models the dimensionless coordinate $x^{\prime}$ is defined as the distance from the potential centre. In this paper, it is also assumed to be a position variable from the potential centre along the major axis since we are fitting along the major axis only. In order to construct a fit for a galaxy, we have to use the linear transformations (14) to convert the dimensionless model variables $\hat{\sigma}, \hat{v}$ and $x^{\prime}$ into dimensional model variables $\sigma, v$ and $r$, which can be compared with the corresponding observations.

From Eq. (14), the Gauss-Hermite coefficients $h_{3}$ and $h_{4}$ in the models can be rewritten as

$$
h_{3}=h_{3}\left(x^{\prime}\right)=h_{3}\left(r / r_{J}\right), h_{4}=h_{4}\left(x^{\prime}\right)=h_{4}\left(r / r_{J}\right) \text {; }
$$

and the dimensional model variables $\sigma$ and $v$ can be regarded as functions of the distance $r$, i.e.,

$$
v=u \hat{v}\left(r / r_{J}\right), \sigma=u \hat{\sigma}\left(r / r_{J}\right)
$$

Once the length and velocity scales $r_{J}$ and $u$ have been determined, the physical parameters of the models can be found from Eq. (14), in order to describe the physical scales of the galaxy. In order to determine the length and velocity scales $r_{J}$ and $u$, we have to use the weighted least squares method to construct a function. Then the two scales $r_{J}$ and $u$ can be obtained by minimizing the function in the physical domain. The detailed process of obtaining the length and velocity scales $r_{J}$ and $u$ is presented below.

Observations corresponding to the Gauss-Hermite fit parameters for any galaxy are discrete. Assume that there are a sequence of observations [say, $\sigma_{i}, v_{i}, h_{3 i}, h_{4 i}$ ] with errors $\left(\delta \sigma_{i}, \delta v_{i}, \delta h_{3 i}, \delta h_{4 i}\right)$, corresponding to the Gauss-Hermite fit parameters, at distances $r_{i}(i=1,2, \cdots, n)$. Let $f\left(r_{J}\right)$ and $g\left(r_{J}, u\right)$ be given by the following two functions:

$$
f\left(r_{J}\right)=\sum_{i=1}^{n}\left(w_{3 i}\left|h_{3}\left(r_{i} / r_{J}\right)-h_{3 i}\right|^{2}+w_{4 i}\left|h_{4}\left(r_{i} / r_{J}\right)-h_{4 i}\right|^{2}\right)
$$

and

$$
g\left(r_{J}, u\right)=\sum_{i=1}^{n}\left(w_{v i}\left|u \hat{v}\left(r_{i} / r_{J}\right)-v_{i}\right|^{2}+w_{\sigma i}\left|u \hat{\sigma}\left(r_{i} / r_{J}\right)-\sigma_{i}\right|^{2}\right),
$$

where the weights $w_{3 i}, w_{4 i}, w_{v i}$ and $w_{\sigma i}$ are related to the observational errors. Thus the length and velocity scales $r_{J}$ and $u$ can be determined by minimizing $f\left(r_{J}\right)+g\left(r_{J}, u\right)$.

The scale lengths $r_{J}$ for the arguments $v$ and $\sigma$ are the same as those for the arguments $h_{n}$. The scale lengths are different from the effective radius $R_{e}$ or core radius $R_{c}$. Here, the effective radius $R_{e}$ is defined to be the radius of the isophote containing half of the total luminosity and the core radius $R_{c}$ is where the surface brightness has fallen to half of its central value. It is found that the scale length $r_{J}$ is about half that of the core radius [i.e. $\left.r_{J} \simeq R_{c} / 2\right]$ for NGC 2974 . 
Generally, the weights $w_{3 i}, w_{4 i}, w_{v i}$ and $w_{\sigma i}$ are chosen as follows.

$$
\begin{aligned}
& w_{3 i}=\frac{e-e^{\delta h_{3 i} / h_{3 m}}}{e-1} e^{-4 \delta h_{3 i} / h_{3 m}}, \quad w_{4 i}=\frac{e-e^{\delta h_{4 i} / h_{4 m}}}{e-1} e^{-4 \delta h_{4 i} / h_{4 m}}, \\
& w_{v i}=\frac{e-e^{\delta v_{i} / v_{m}}}{e-1} e^{-4 \delta v_{i}}, \quad w_{\sigma i}=\frac{e-e^{\delta \sigma_{i} / \sigma_{m}}}{e-1} e^{-4 \delta \sigma_{i}} .
\end{aligned}
$$

We take $h_{3 m}=h_{4 m}=0.2$ and $v_{m}=\sigma_{m}=300 \mathrm{kms}^{-1}$, since the range of the measured velocity dispersions is usually $100-300 \mathrm{kms}^{-1}$ (Gerhard 1993a; Bender et al. 1994) and the third-order and fourth-order GaussHermite coefficients range from -0.2 to 0.2 (Gerhard 1993a; Surma and Bender 1995) for elliptical galaxies. Our choice of the weights (19) and (20) is based on the following three reasons. First, the weights should be equal to zero when the observational errors attain their corresponding maximal values ( 0.2 for the Gauss-Hermite moments and $300 \mathrm{kms}^{-1}$ for rotation velocities and velocity dispersions), and unity when the observational errors are zero. Secondly, the weights should decrease with the observational errors. Thirdly, the weights should be continuous functions, for convenience of calculation.

\section{Determination of the Total Mass of the Model}

It has been shown in Sect. 3 that the model variables can be related to the corresponding observations of the galaxy if the length and velocity scales $r_{J}$ and $u=\left(G M / r_{J}\right)^{1 / 2}$ are known. In this section we show how to use the two scales $r_{J}$ and $u$ to calculate the finite total mass $M$ of the oblate model defined by Eqs. (1) and (2).

As mentioned in Sect. 3, the distance $r$ from the galactic centre is generally given in arcsec (Cinzano and van der Marel 1994; Bender et al. 1994) and the model variable $x^{\prime}$ is dimensionless. For convenience when comparing the model variables with the corresponding observations, the two scales $r_{J}$ and $u$ in (14) are given in arcsec and $\mathrm{kms}^{-1}$ respectively. Suppose that $d_{0}$ is the distance from this galaxy to the observer in units of Mpc. Assume that $p$ (in arcsec) is the length scale $r_{J}$ of a model. If the Gauss-Hermite fit parameters for the model with the two scales $r_{J}$ and $u$ can fit the corresponding observations of a galaxy following minimization of a function with (17) and (18), then $p$ and $u$ can be obtained by comparing the model variables $\left(x^{\prime}, \hat{v}, \hat{\sigma}, h_{3}, h_{4}\right)$ with the corresponding observed quantities $\left(r, v, \sigma, h_{3}, h_{4}\right)$ by use of the linear transformation (14). On the other hand, the length and velocity units of the model are $r_{J}$ and $\left(G M / r_{J}\right)^{1 / 2}$. Since the two scales $r_{J}$ and $u$, obtained by comparing the Gauss-Hermite fit parameters of the models with the corresponding observations, are the same as the length and velocity units of the model respectively, we have

$$
r_{J}=d_{0}\left(\frac{\pi p}{180 \times 3600}\right)
$$

and

$$
\left(G M / r_{J}\right)^{1 / 2}=u .
$$

Indeed, (21) is also a transformation from a distance unit of 1 arcsec to a unit of $1 \mathrm{Mpc}$. Since it has been shown by Jiang (2000) that the total mass of the model is finite and equal to $M$, it is known from Eqs. (21) and (22) that the total mass $M$ of the model is

$$
M=\frac{u^{2} r_{J}}{G}=\frac{u^{2} d_{0}}{G}\left(\frac{\pi p}{180 \times 3600}\right) .
$$

\section{Fitting of Oblate Jaffe Models}

In this section we obtain a good fit of an oblate Jaffe model by mimicking the image and ellipticity of the galaxy NGC 2974, and comparing the Gauss-Hermite fit parameters of the model with the corresponding observations of the galaxy. But, it is finally found that the derived finite total mass of the model is quite close to the total luminous mass of this galaxy.

It has been mentioned in Sect. 3 that the oblate model defined by Eqs. (1) and (2) with physical parameters $\left(c, d, r_{J}\right)$ first has to be nondimensionalized to the dimensionless variables $\hat{c}$ and $\hat{d}$, which are given by analysing the ellipticity and contours of the image of the galaxy, so that the model can mimic the image of the galaxy. NGC 2974 is classified as E4, and the flattening of the contours in B-R images of NGC 2974 (Kim 1989) suggests that the inclination angle of the minor axis to the line of sight of NGC 2974 is about $60^{\circ}$ (Cinzano 


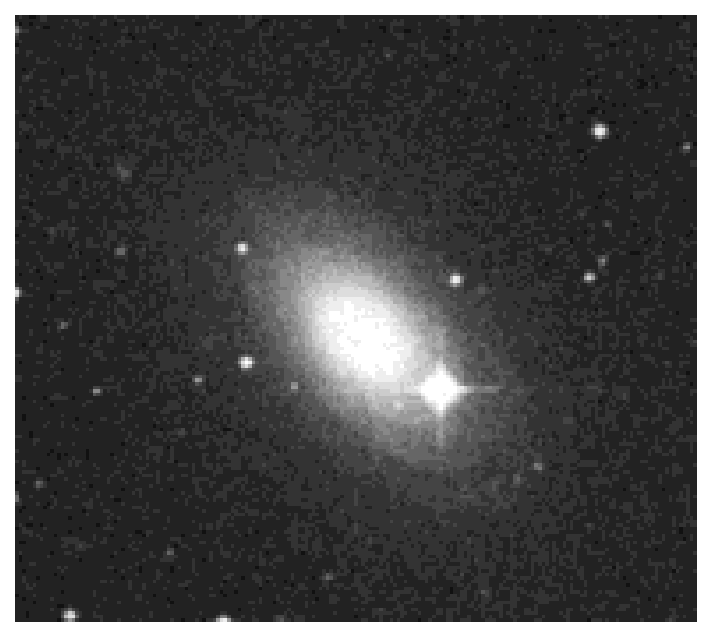

Fig. 1 The image of NGC 2974 obtained from NASA/IPAC Extragalactic Database (NED).

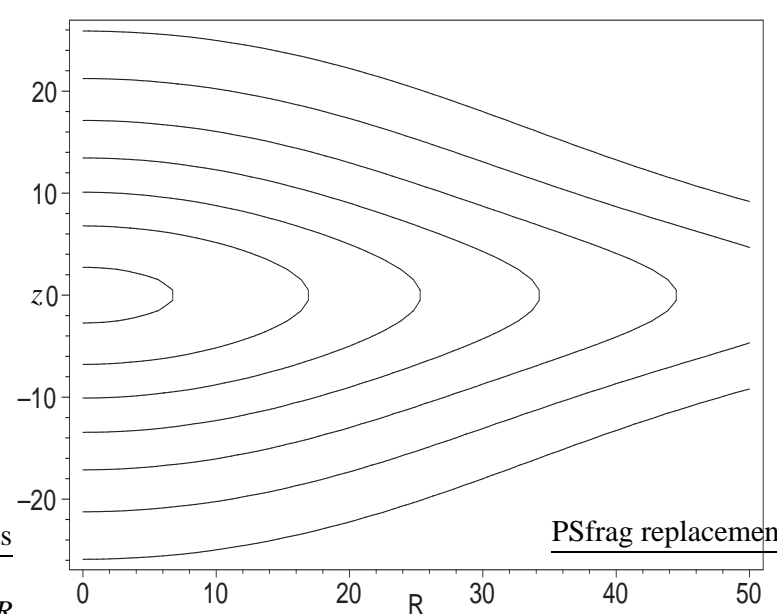

Fig. 2 The contours of the mass density $\rho\left(R^{2}, z\right)$ in (2) for $c=$ $8.43 r_{J}$ and $d=15 r_{J}$. Successive contour levels differ by factors of 0.5 .

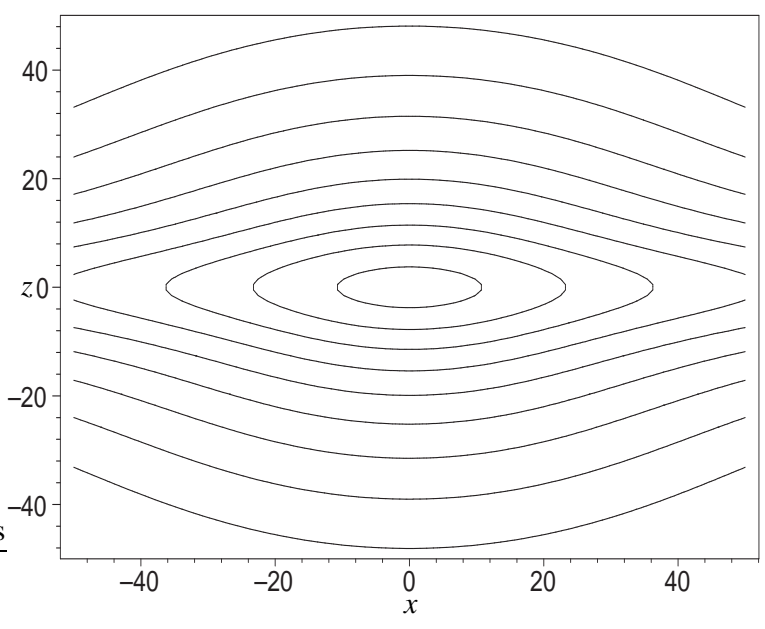

Fig. 3 The contours of the edge-on projected surface density generated by $\rho\left(R^{2}, z\right)$ in (2) for $c=8.43 r_{J}$ and $d=15 r_{J}$. Successive contour levels differ by factors of 0.5 .

and van der Marel 1994). Thus we examine oblate models of ellipticity E4 with an inclination angle of $60^{\circ}$ to describe NGC 2974. On the other hand, it is easy to see from Eq. (3) that the dimensionless model is of type E4 when $\hat{c}=8.43$ and $\hat{d}=15$ [that is, $c=8.43 r_{J}$ and $d=15 r_{J}$ ]. Of course, there are an infinity of solutions $(\hat{c}, \hat{d})$ of Eq. (3) that make the dimensionless model be of type $\mathrm{E} 4$, and $(\hat{c}, \hat{d})=(8.43,15)$ is just one of these, and is such that not only is the ellipticity of the model close enough to that of the galaxy NGC 2974 but also the contours of the projected surface density of the model are quite similar to those of the image of the galaxy. That is, it can be applied to simulate the image of the galaxy. We find the image of the galaxy from the NASA/IPAC Extragalactic Database and plot the contours of the mass density of this model and its edge-on projected surface density. Here, Fig. 1 shows the image of NGC 2974, and Fig. 2 and Fig. 3 display the contours of the mass density and its edge-on projected surface density, respectively. In Fig. 2 and Fig. 3, successive contour levels differ by factors of 0.5 , from the potential centre to the outskirts, and the isophotes get more flattened outwards along the major axis. This illustates that the isophotes of the model become more disky outwards, as the mass density drops off slowly like $r^{-3}$ along the major axis.

Once the dimensionless model is determined by using the image and ellipticity of the galaxy, the GaussHermite fit parameters at any inclination angle for the model can be calculated, by using the maximum entropy principle or assuming the isotropy (or anisotropy) of the model (Jiang 2000). Then we use a weighted least squares algorithm, as mentioned in Sect. 3, to compare the Gauss-Hermite fit parameters of the model with the corresponding observations of NGC 2974. Since the model is oblate, we can assume arbitrarily that the model is isotropic. Minimization of the function $f\left(r_{J}\right)+g\left(r_{J}, u\right)$ shows that an oblate model given by Eqs. (1) and (2) with $c=8.43 r_{J}$ and $d=15 r_{J}$ can be used to determin the Gauss-Hermite fit parameters of NGC 2974, with an inclination angle of $60^{\circ}$. Further, the length and velocity scales are $r_{J}=0.55 \operatorname{arcsec}$ (or say, $p=0.55 \operatorname{arcsec}$ ) 

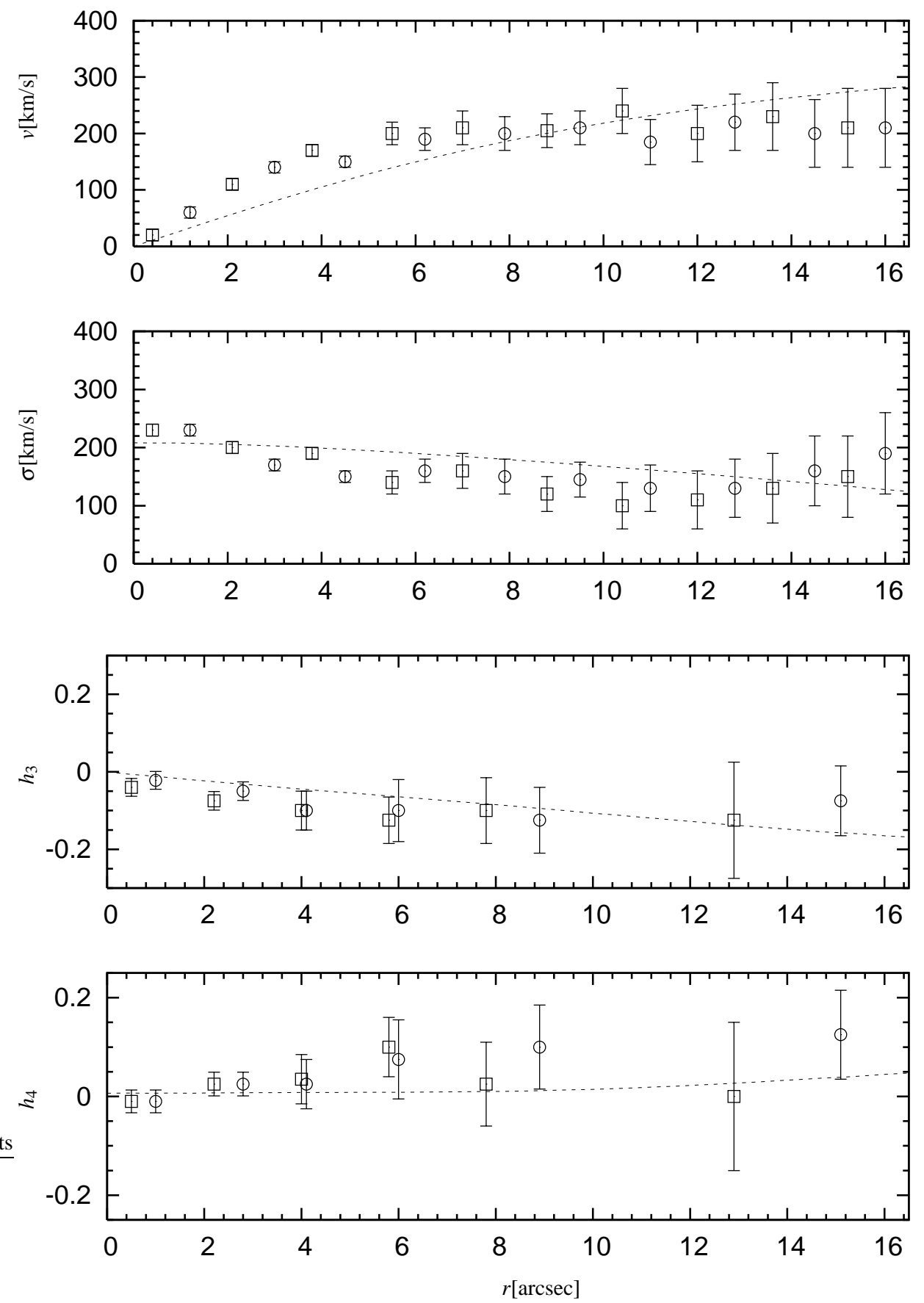

Fig. 4 Gauss-Hermite fit parameters for NGC 2974: the error bars represent the observations of Cinzano and van der Marel (1994), the broken curves come from the oblate model defined by Eqs. (1) and (2) with $c=8.43 r_{J}$ and $d=15 r_{J}$, assuming isotropy with the choice of $r_{J}=0.55$ arcsec and $u=3300 \mathrm{kms}^{-1}$. The inclination angle of the minor axis to the line of sight is $60^{\circ}$.

and $u=3300 \mathrm{kms}^{-1}$ respectively. Fig. 4 shows this fit. Our diagnostic or criterion for deciding the quality of the fit is that the weighted least squares fit is regarded as good if the mean absolute error values of the velocity $v$, the dispersion $\sigma$, and the Gauss-Hermite coefficients $h_{3}$ and $h_{4}$, between the model and the observations, are less than $15 \mathrm{kms}^{-1}, 15 \mathrm{kms}^{-1}, 0.01$ and 0.01 , respectively. For this model, the mean absolute error values between these fit parameters $\left(v, \sigma, h_{3}, h_{4}\right)$ and their corresponding observations are, respectively, $12.75 \mathrm{kms}^{-1}$, $12.00 \mathrm{kms}^{-1}, 0.005$ and 0.004 . Therefore this fitting can be regared as good.

The error bars in Fig.44represent the major axis Gauss-Hermite fit parameters of NGC 2974 [see Cinzano and van der Marel 1994, figure 1 or figure 5(a)]. These observations are obtained by analysing the galaxy spectrum data with the modified Fourier fitting method developed independently by van der Marel and Franx (1993) and 
Gerhard (1993b). This data analysis is used to determine the derivations of the velocity distribution curve from a Gaussian.

The galaxy spectrum is assumed to be the convolution of a template spectrum and the velocity distribution curve. The velocity distribution curve is expanded as the Gauss-Hermite series of the five Gauss-Hermite fit parameters, which are defined by (10) with (11) and (12). The parameters are determined by $\chi^{2}$ fitting in Fourier space of the broadened template spectrum to the galaxy spectrum. The finite spectral resolution of the instrument needs a high- and a low- wavenumber cut-off. To obtain parameters $(\gamma, v, \sigma)$ from the galaxy spectra, a Gaussian velocity profile can be fitted to the spectra. This requires less rebinning of the data in the spatial direction. To get the parameters $\left(h_{3}, h_{4}\right)$, the parametrization (12) can be fitted to the data.

The galaxy spectra are in fact the data that are produced by the standard reduction of stellar spectra with one spectrum of high signal-to-noise ratio for each star. This data reduction is done as follows. First, wavelength calibration is done using He-Ar arc lamp spectra, and so is sky subtraction using the available data at the ends of the slit. After that, frames should be flat-fielded and cleaned from cosmic rays and CCD defects. Finally, the galaxy spectra are rebinned in the spatial direction, with emission lines of $4959 \AA, 5007 \AA$ and $5200 \AA$ interpolated over, in order to increase the signal-to-noise ratio.

The stellar spectra are obtained by using a spectrograph equipped with CCD. To do this, one has to consider the exposures of the spectrograph, the scale of the rebinned stellar spectra, the sky seeing, the dispersion of the grating, the range of the spectra, the width of the slits, and so on. The spectroscopic data of slits along the major axis $\left(\mathrm{PA}=225^{\circ}\right)$ of NGC 2974 are first taken through two separate 45-min exposures that are reduced separately and added later. The stellar spectra are then rebinned at the telescope over 3 pixels in the spatial direction and so have a scale of $1.65 \mathrm{arcsec} / \mathrm{pixel}$. The seeing during the observations is about 1.9 arcsec FWHM. The grating used has a dispersion of $0.89 \AA$ /pixel. The chosen spectral range is from 4750 to $5600 \AA$, centred at about 5175 $\AA$. The slit width is 1.51 arcsec so that the instrument velocity resolution is $45 \mathrm{~km} / \mathrm{s}$.

The broken curves in Fig. 4 are those for the parameters of the oblate model mentioned above. The curve of $v$ fits well the velocity of NGC 2974 at radii between 7 to 14 arcsec, although inside a radius of about 7 arcsec, the model velocity is lower than that observed, and is higher at distances larger than about $14 \operatorname{arcsec}$. The curve of $\sigma$ lies within the corresponding error bars in the region from 11 to 16 arcsec, but it is lower inside a radius of about 2 arcsec and higher in the range from 2 to 11 arcsec. As the radius increases, the dispersion of the model always decreases; but the dispersion of NGC 2974 tends to increase with radius from about 12 arcsec. The graphs of the parameters $h_{3}$ and $h_{4}$ are the best fit for the four broken curves in Fig. 4, they are within, or outside but very close to their corresponding error bars. In a word, the fit of the four parameters in Fig. 4 is credible, and so it is a good example to demonstrate our approach of modelling elliptical galaxies dynamically.

The Virgocentric flow model of Aaronson et al. (1982) gives $d_{0}=22.5 \mathrm{~h}^{-1} \mathrm{Mpc}$. Eq. (21), when $p=0.55$ arcsec and $d_{0}=22.5 \mathrm{Mpc}$, gives $r_{J}=0.06 \mathrm{kpc}$. Then, by using (23), the total mass $M$ of the model, given by $c=8.43 r_{J}$ and $d=15 r_{J}$, can be calculated as $M=1.52 \times 10^{11} h^{-1} \mathrm{M}_{\odot}$. This mass is very close to the total luminous mass of NGC 2974 mentioned in Sect. 1 [i.e., that predicted by Cinzano and van der Marel (1994)]. This is consistent with the total mass $M$ of the models only reflecting the total luminous mass. This is partly because we choose the model only in terms of the image and ellipticity of the luminosity of the galaxy, and partly because the influence of the dark halo in NGC 2974 on the Gauss-Hermite fit parameters of the galaxy is negligible within a radius of $0.5 R_{\mathrm{e}}$, and the fitting of Gauss-Hermite fit parameters given in Fig. 4 is made just within this region where the dynamical influence of the dark halo is negligible (cf. also Cinzano and van der Marel 1994). But the radius of the isophote containing half of the total mass of this model is 18.59 arcsec, which is less than the effective radius of NGC 2974. This also implies that the dark matter halo extends to the central area of the ellipsoidal galaxy NGC 2974. It is worth mentioning that Weijimans et al. (2008) recently found that within $5 R_{\mathrm{e}}$ at least 55 per cent of the total mass is dark for the early-type galaxy NGC 2974.

Now we consider another model of type E4. When $(\hat{c}, \hat{d})=(1.26,1)$ [that is, $c=1.26 r_{J}$ and $d=r_{J}$ ], it can be found from Eq. (3) that the dimensionless model is of type E4. Fig. 5] and Fig. 6 show the contours of the density of this model, and its edge-on projected surface density, respectively. They are similar to the corresponding figures for $c=8.43 r_{J}$ and $d=15 r_{J}$ (see Fig. 2 and Fig. 3), respectively. Similarly, in order to fit the galaxy Gauss-Hermite parameters, the length and velocity scales $\left(r_{J}, u\right)$ can be calculated as follows: $r_{J}=3.2$ arcsec (or say, $p=3.2 \mathrm{arcsec}$ ) and $u=1110 \mathrm{kms}^{-1}$. Fig. 7 shows a Gauss-Hermite parameter fit for NGC 2974. It can thus be found that the total mass $M$ of this model is equal to $1.0 \times 10^{11} h^{-1} \mathrm{M}_{\odot}$. This mass is less than that of the model with $(\hat{c}, \hat{d})=(8.43,15)$, and it is farther away from the total luminous mass of NGC 2974 than that of the latter. On the other hand, in Fig. 4 and Fig. 7, the corresponding broken curves of the two models are almost similar and their difference is very small. Our criterion of determining a better model is thus as follows: the closer the total mass of the model is to the mass of NGC 2974, the better the model is. Therefore, we believe that the model with $(\hat{c}, \hat{d})=(8.43,15)$ is a better fit for NGC 2974 than that with $(\hat{c}, \hat{d})=(1.26,1)$, although the velocity $v$ fitting in Fig. 4 is worse than that in Fig. 7 . For the model with $(\hat{c}, \hat{d})=(1.26,1)$, the mean absolute 


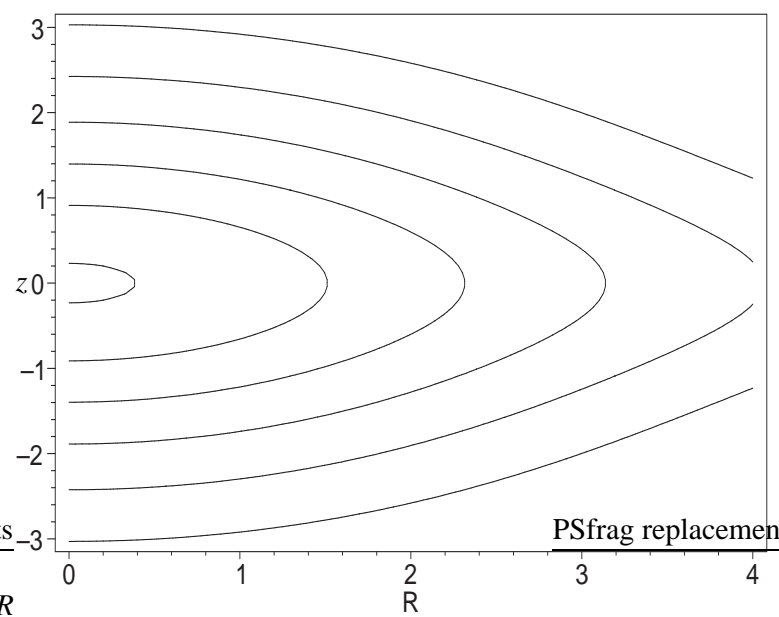

Fig. 5 As Fig. 2 but for $c=1.26 r_{J}$ and $d=r_{J}$.

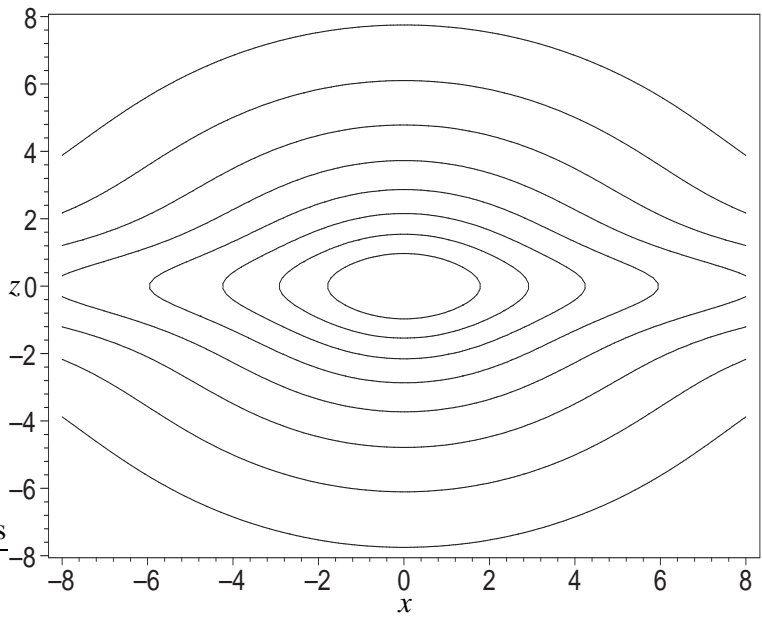

Fig. 6 As Fig. 3 but for $c=1.26 r_{J}$ and $d=r_{J}$.

error values between the parameters $\left(v, \sigma, h_{3}, h_{4}\right)$ and their corresponding observations can be calculated as 8.75 $\mathrm{kms}^{-1}, 14.00 \mathrm{kms}^{-1}, 0.0085$ and 0.004 , respectively. Of course, the fitting in Fig. 7 is also a good fit.

In Fig. 4 and Fig. 7 the systemic velocity of the two models is monotonic while the corresponding galaxy observation values flatten out. This is a shortcoming of our models. It might result from our assumption of isotropy, which would in turn imply that the galaxy is very anisotropic.

It is further found from our computations that models of type $\mathrm{E} 4$ have similar profiles of the Gauss-Hermite fit parameters $\left(v, \sigma, h_{3}, h_{4}\right)$. Generally, the smaller the ratio $c / d$, the better are the models of the type corresponding to that of the galaxy. Hence, there might be other better fit models for NGC 2974, although we cannot discuss these possibilities here.

\section{Conclusions}

Dynamical models are generated from both the gravitational potentials and the distributional functions of the stellar systems, based on dynamical principles. Therefore the analysis of the dynamical model described above can be summarised into two steps. The first is to search for a dimensionless flattened Jaffe model whose projected surface density generated from its gravitational potential can be used to fit the actual surface brightness of the galaxy. To do this, the ellipticity of the chosen model must be close enough to that of the image of the galaxy that the contours of the model are similar to those of the image of the surface brightness of the galaxy. Of course, the contours of the projected surface density of the model must become more disky outwards as its corresponding density drops off slowly along the major axis. The second step is to determine the length and velocity scales of the dynamical model by comparing the model variables with the corresponding observational scales (i.e. by comparing their Gauss-Hermite fit parameters which are needed for quantifying their velocity distribution curves). The Gauss-Hermite fit parameters of the model are obtained from its distributional functions.

The ellipticity of the model can be determined by the ratio of $R$-axis to $z$-axis extent of the contours of the density $\rho\left(R^{2}, z\right)$ near the origin. In order to compare the fit parameters $\left(\hat{v}, \hat{\sigma}, h_{3}, h_{4}\right)$ of the model with the corresponding observations, the ellipticity of the model should be the same as that of the B-R images of the considered galaxy. The ellipticity of the galaxy being considered can be obtained from observations. By using the ellipticity obtained, a model can be chosen to fit the image of the surface brightness of the galaxy being considered. For example, for the oblate model, $\hat{c}$ can be obtained from Eq. (3) if it is assumed that $c=\hat{c} r_{J}$ and that $d=r_{J}$. The model has first to be transformed into a dimensionless model in terms of $\hat{c}$, and then the fit parameters $\left(\hat{v}, \hat{\sigma}, h_{3}, h_{4}\right)$ of the dimensionless model have to be calculated. It is necessary to make an assumption about the anisotropy of the models; we choose an isotropic model.

Comparison with observations requires relations between the observational scales and the model variables. The relations are given by the linear transformation (14). This transformation can be determined if $r_{J}$ and $\left(G M / r_{J}\right)^{1 / 2}$ are known. Therefore, comparing the model variables with observations by use of the linear transformation, a good pair of values of $r_{J}$ and $\left(G M / r_{J}\right)^{1 / 2}$ can be chosen, such that the model variables fit the corresponding observations.

The total mass of the flattened Jaffe models can be shown to be $M$. Thus the values of $r_{J}$ and $\left(G M / r_{J}\right)^{1 / 2}$ finally lead to the total mass $M$ of the model. Since the model only reflects the B-R images of the galaxy being considered, namely NGC 2974, the total mass $M$ of the model is obviously less than the total mass of this galaxy 

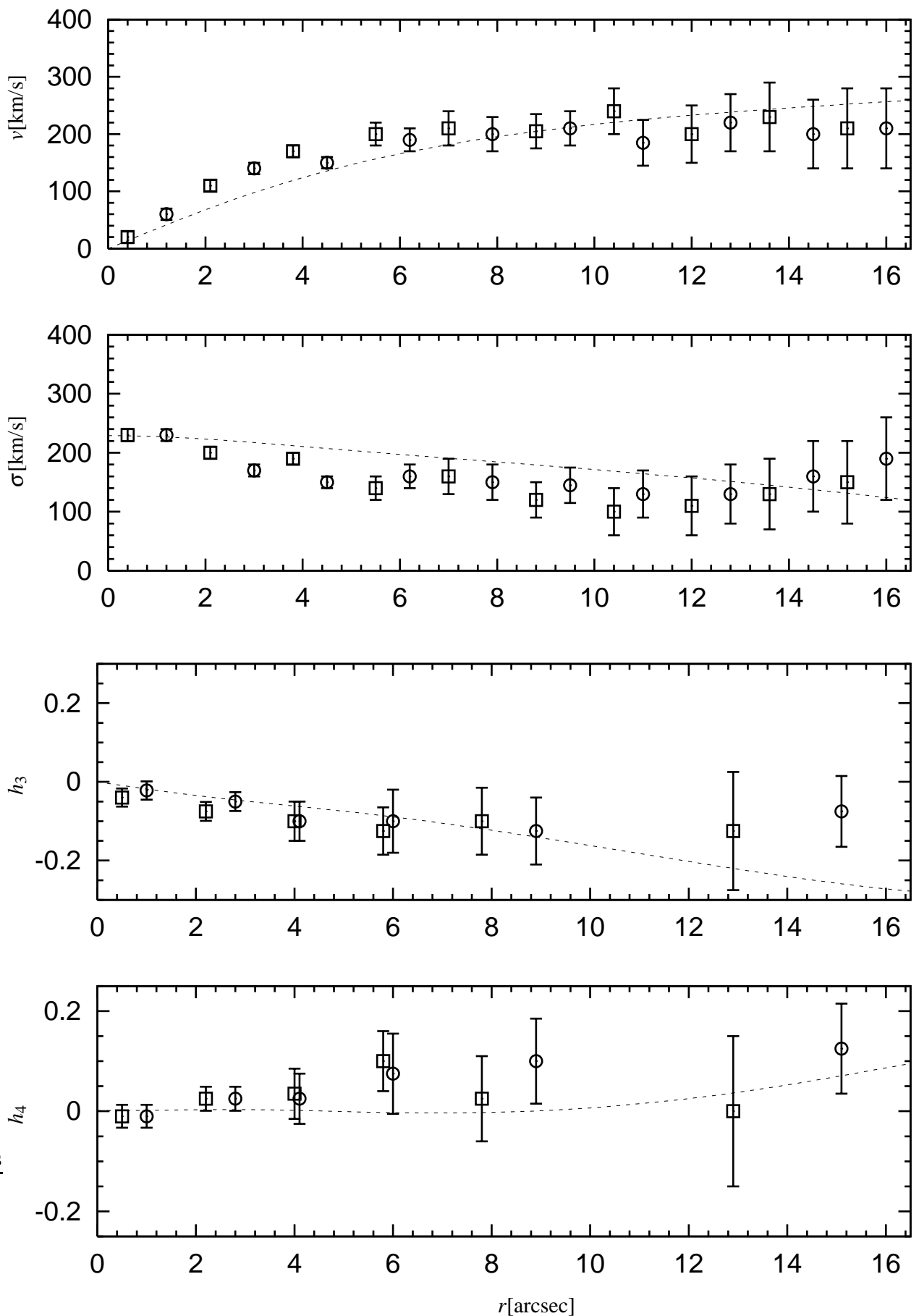

Fig. 7 As Fig. 4 but the broken curves are obtained for $c=1.26 r_{J}$ and $d=r_{J}$, assuming isotropy, with the length and velocity scales $r_{J}=3.2$ arcsec and $u=1110 \mathrm{kms}^{-1}$.

and so it reflects the total luminous mass of this galaxy. Additionally, the dynamical influence of dark matter in NGC 2974 on the Gauss-Hermite fit parameters of the galaxy observed is negligible inside a radius of $0.5 R_{\mathrm{e}}$.

For a very flattened model, the two values of $r_{J}$ and $\left(G M / r_{J}\right)^{1 / 2}$ can be also determined by comparing the circular velocities generated from the potential of the model with data of the rotation velocities of the very flattened galaxies observed. This is the customary approach of fitting the projected surface density of the model to the observed surface brightness of the galaxies. This result must be the same as that obtained by comparing the Gauss-Hermite fit parameters, if the model is a very good fit. 
The resulting fit in Fig. 4 is satisfactory, and so it is a good example to illustrate our method for constructing such models. And, up to now, no other plausible alternatives have been found for choosing a suitable flattened Jaffe model for the elliptical galaxy NGC 2974.

Acknowledgements ZJ is supported by NSFC 10271121 and by joint grants of NSFC 10511120278/10611120371 and RFBR 0402-39026. The considerable assistance given by members of the High Performance Computing Service of Manchester Computing is acknowledged. ZJ is indebted to Dr Paul Stewart, Dr Richard James, Dr N. Wyn Evans and Dr R. P. van der Marel for helpful comments. ZJ thanks Professor Joan Walsh for her advice on data fitting and Professor Christopher Hunter for his advice on Hunter and Qian's algorithm (1993). ZJ is also very grateful to Professor Konstantin Kholshevnikov, Sergei Kutuzov, Vadim Antonov and Dr Leonid Ossipkov for their valuable discussions on this work. ZJ thanks Dr David Moss very much for his providing a lot of scientific ideas in this work. Finally, ZJ would like to thank the referee of this paper for his/her helpful comments and suggestions.

\section{References}

Aaronson M., Huchra J. P., Mould J., Schechter P. L., Tully R. B.: The velocity field in the local supercluster. Astrophysical Journal, 258, 64-76 (1982).

Baes M., Dejonghe H.: A completely analytical family of dynamical models for spherical galaxies and bulges with a central black hole. Monthly Notices of the Royal Astronomical Society, 351, 18-30 (2004).

Baes M., Dejonghe H., Buyle P.: The dynamical structure of isotropic spherical galaxies with a central black hole. Astronomy \& Astrophysics, 432, 411-422 (2005)..

Bender R., Saglia R. P., Gerhard O. E.: Line-of-Sight Velocity Distributions of Elliptical Galaxies. Monthly Notices of the Royal Astronomical Society, 269, 785-813 (1994).

Bennett C. L., et al.: First-Year Wilkinson Microwave Anisotropy Probe (WMAP) Observations: Preliminary Maps and Basic Results. The Astrophysical Journal Supplement Series, 148, 1-27 (2003).

Bennett C. L., et al.: The Microwave Anisotropy Probe Mission. The Astrophysical Journal, 583, 1-23 (2003).

Binney J., Tremaine S.: Galactic Dynamics. Princeton Univ., Princeton (1987).

Canizares C. R., Fabbiano G., Trinchieri G.: Properties of the X-ray emitting gas in early-type galaxies. Astrophysical Journal, 312, 503-513 (1987).

Cappellari M., Emsellem E.: Parametric Recovery of Line-of-Sight Velocity Distributions from Absorption-Line Spectra of Galaxies via Penalized Likelihood. The Publications of the Astronomical Society of the Pacific, 116, 138-147 (2004).

Cinzano P., van der Marel R. P.: Observations and Dynamical Modelling of the e4 Galaxy NGC2974 - Evidence for an Embedded Stellar Disc. Monthly Notices of the Royal Astronomical Society, 270, 325-340 (1994).

Davies R. L., Burstein D., Dressler A., Faber S. M., Lynden-Bell D., Terlevich R., Wegner G.: Spectroscopy and photometry of elliptical galaxies. II - The spectroscopic parameters. Astrophysical Journal, 64, 581-600 (1987).

Dehnen W.: A family of potential-density pairs for spherical galaxies and bulges. Monthly Notices of the Royal Astronomical Society, 265, 250-256 (1993)

Dejonghe H.: Stellar dynamics and the description of stellar systems. Physics Reports, 133, 217-313 (1986).

Demoulin-Ulrich M. H., Butcher H. R., Boksenberg A.: Extended gaseous emission in normal elliptical galaxies. Astrophysical Journal, 285, 527-546 (1984).

de Vaucouleurs G., de Vaucouleurs A., Faber S. M., Jr Buta R. J., Paturel H. G., Fouqué P.: Reference Catalogue of Bright Galaxies, Springer-Verlag, New York, 3rd (1991).

Emsellem E., Goudfrooij P., Ferruit P.: A two-arm gaseous spiral in the inner 200 pc of the early-type galaxy NGC 2974: signature of an inner bar. Monthly Notices of the Royal Astronomical Society, 345, 1297-1312 (2003).

Evans N. W.: The power-law galaxies. Monthly Notices of the Royal Astronomical Society, 267, 333-360 (1994).

Forman W., Jones C., Tucker W.: Hot coronae around early-type galaxies. Astrophysical Journal, 293, 102-119 (1985).

Gallouet L., Heidmann N., Dampierre F.: Optical positions of bright galaxies. III. Astronomy \& Astrophysics Supplement Series, 19, 1-19 (1975).

Gerhard O. E.: Elliptical Galaxies, in Lecture notes in physics, Vol. 433 (1993a).

Gerhard O. E.: Line-of-sight velocity profiles in spherical galaxies: breaking the degeneracy between anisotropy and mass. Monthly Notices of the Royal Astronomical Society, 265, 213-230 (1993b).

Gerhard O. E., Jeske G., Saglia R. P., Bender R.: Breaking the degeneracy between anisotropy and mass: the dark halo of the E0 galaxy NGC 6703. Monthly Notices of the Royal Astronomical Society, 295, 197-215 (1998).

Halliday C., Davies R. L., Kuntschner H., Birkinshaw M., Bender R., Saglia R. P., Baggley G.: Line-of-sight velocity distributions of low-luminosity elliptical galaxies. Monthly Notices of the Royal Astronomical Society, 326, 473-489 (2001).

Hunter C., Qian E.: Two-integral distribution functions for axisymmetric galaxies. Monthly Notices of the Royal Astronomical Society, 262, 401-428 (1993).

Jaffe W.: A simple model for the distribution of light in spherical galaxies. Monthly Notices of the Royal Astronomical Society, 202, 995-999 (1983).

Jiang Z.: Flattened Jaffe models for galaxies. Monthly Notices of the Royal Astronomical Society, 319, 1067-1078 (2000).

Jiang Z., Fang D., Liu H., Moss D.: General flattened Jaffe models for galaxies. AMS/IP Studies in Advanced Mathematics, 29, Geometry and Nonlinear Partial Differential Equations, p. 31-37 (2002).

Jiang Z., Fang D., Moss D.: Axisymmetric models for galaxies by equipotential and equidensity methods. Proceedings of the Sixth Conference of China Society for Industry and Applied Mathematics, Research Information Ltd, Hertfordshire, UK, p. 79-83 (2002)

Jiang Z., Moss D.: Prolate Jaffe models for galaxies. Monthly Notices of the Royal Astronomical Society, 331, 117-125 (2002).

Jiang Z., Ossipkov L. P.: Flattened $\gamma$ models for galaxies. Astronomical and Astrophysical Transactions, 25, 213-216 (2006).

Jiang Z., Ossipkov L. P.: Anisotropic distribution functions for spherical galaxies. Celestial Mechanics and Dynamical Astronomy, 97, 249-265 (2007a).

Jiang Z., Ossipkov L. P.: Two-integral distribution functions for axisymmetric systems. Monthly Notices of the Royal Astronomical Society, 379, 1133-1142 (2007b).

Jiang Z., Ossipkov L. P.: Two-integral distribution functions for axisymmetric stellar systems with separable densities. Monthly Notices of the Royal Astronomical Society, 382, 1971-1981 (2007c).

Kim D. W.: Interstellar matter in early-type galaxies - Optical observations. Astrophysical Journal, 346, 653-674 (1989). 
Kim D. W., Guhathakurta P., van Gorkom J. H., Jura M., Knapp G. R.: H I observations of the elliptical galaxies NGC 2974 and NGC 5018. Astrophysical Journal, 330, 684-694 (1988).

Krajnović D., Cappellari M., Emsellem E., McDermid R. M., de Zeeuw P. T.: Dynamical modelling of stars and gas in NGC 2974: determination of mass-to-light ratio, inclination and orbital structure using the Schwarzschild method. Monthly Notices of the Royal Astronomical Society, 357, 1113-1133 (2005).

Kronawitter A., Gerhard O., Saglia R. P., Bender R.: Dynamical analysis of elliptical galaxy halos. Galaxy Dynamics, ASP Conference Series, 182, 441-442 (1999).

Kutuzov S. A., Ossipkov L. P.: A generalized model for the three-dimensional gravitational potential of stellar systems. Pis'ma v Astronomicheskij Zhurnal, 57, 28-37 (1980) (English translations: Soviet Astronomy Letters, 24, 17-22, (1981)).

Kuzmin G. G., Veltmann Ü.-I.K.: Hydrodynamic models of spherical stellar systems. W. Struve Tartu Astrofüüs. Obs. Publ., 36, 3-47 (1967a).

Kuzmin G. G., Veltmann Ü.-I.K.: Lindblad diagram and isochronic models. W. Struve Tartu Astrofüüs. Obs. Publ., 36, 470-507 (1967b).

Kuzmin G. G., Veltmann Ü.-I.K.: Generalized isochrone models for spherical stellar systems. Dynamics of Galaxies and Star Clusters, Nauka, Alma-Ata, 82-87 (1973) (English translations: Galactic Bulges (IAU Symp. 153), Kluwer, Dordrecht, 363-366 (1993)).

Kuzmin G. G., Veltmann Ü.-I.K., Tenjes P. L.: Quasi-isothermal models of spherical stellar systems. Application to the galaxies M 87 and M 105. W. Struve Tartu Astrofüüs. Obs. Publ., 51, 232-242 (1986).

Ossipkov L. P.: Some problems of the theory of self-consistent models for star clusters. Star Clusters, Urals Univ. Press, Sverdlovsk, 72-89 (1979a).

Ossipkov L. P.: Spherical systems of gravitating bodies with an ellipsoidal velocity distribution. Pis'ma v Astronomicheskij Zhurnal, 5, 77-80 (1979b) (English translations: Soviet Astronomy Letters, 5, 42-44).

Ossipkov L. P., Jiang Z.: Density asymptotics for infinite gravitating system. Messenger of Saint Petersburg University, Ser. 10, Applied Mathematics, Informatics, Control Processes, Iss. 2, 66-74 (2007a).

Ossipkov L. P., Jiang Z.: Constructing galaxy models with a central density cusp by equipotential method. Messenger of Petersburg University, Ser. 1, Mathematics, Mechanics, Astronomy, Iss. 1, 139-143 (2007b).

Qian E. E., de Zeeuw P. T., van der marel R. P., Hunter C.: Axisymmetric galaxy models with central black holes, with an application to M32. Monthly Notices of the Royal Astronomical Society, 274, 602-622 (1995).

Schwarzschild M.: A numerical model for a triaxial stellar system in dynamical equilibrium. Astrophysical Journal, 232, 236-247 (1979)

Saglia R. P., Kronawitter A., Gerhard O., Bender R.: The orbital structure and potential of NGC 1399. Astronomical Journal, 119, 153-161 (2000).

Saha P.: Designer basis functions for potentials in galactic dynamics. Monthly Notices of the Royal Astronomical Society, 262, 1062-1064 (1993)

Sandage A. R., Tammann G. A.: Revised Shapley-Ames Catalogue of Galaxies, Washington DC: Carnegie Institution of Washington (1981).

Surma P., Bender R.: Relics of dissipational merging and past violent starbursts in elliptical galaxies - the gE galaxy NGC 4365 . Astronomy \& Astrophysics, 298, 405-419 (1995).

Thomas, J. et al.: Dynamical modelling of luminous and dark matter in 17 Coma early-type galaxies. Monthly Notices of the Royal Astronomical Society, 382, 657-684 (2007).

Tremaine S. et al.: A family of models for spherical stellar systems. Astronomical Journal, 107, 634-644 (1994).

van der Marel R. P., Franx M.: A new method for the identification of non-Gaussian line profiles in elliptical galaxies. Astrophysical Journal, 407, 525-539 (1993).

Veltmann Ü.-I.K.: Constructing models for spherical star systems with given space density. Tartu Astron. Obs. Publ., 33, 387-415 (1961)

Veltmann Ü.-I.K.: On phase density of spherical stellar systems. W. Struve Tartu Astrofüüs. Obs. Publ., 35, 5-26 (1965).

Veltmann Ü.-I.K.: Phase space models for star clusters. Star Clusters, Urals Univ. Press, Sverdlovsk, 50-71 (1979).

Veltmann Ü.-I.K.: Gravitational potential, space density and phase density of star clusters. W. Struve Tartu Astrofüüs. Obs. Publ., 48, 232-261 (1981).

Weijmans A., Krajnović D., van de Ven G., Oosterloo T. A., Morganti R., de Zeeuw P. T.: The shape of the dark matter halo in the early-type galaxy NGC 2974. Monthly Notices of the Royal Astronomical Society, 383, 1343-1358 (2008). 